\title{
Chapter 11 \\ From Intuitionism to Many-Valued \\ Logics Through Kripke Models
}

\author{
Saeed Salehi
}

\begin{abstract}
Intuitionistic Propositional Logic is proved to be an infinitely many valued logic by Gödel (Kurt Gödel collected works (Volume I) Publications 1929-1936, Oxford University Press, pp 222-225, 1932), and it is proved by Jaśkowski (Actes du Congrés International de Philosophie Scientifique, VI. Philosophie des Mathématiques, Actualités Scientifiques et Industrielles 393:58-61, 1936) to be a countably many valued logic. In this paper, we provide alternative proofs for these theorems by using models of Kripke (J Symbol Logic 24(1):1-14, 1959). Gödel's proof gave rise to an intermediate propositional logic (between intuitionistic and classical), that is known nowadays as Gödel or the Gödel-Dummett Logic, and is studied by fuzzy logicians as well. We also provide some results on the inter-definability of propositional connectives in this logic.
\end{abstract}

Keywords Intuitionistic propositional logic $\cdot$ Many-Valued logics $\cdot$ Kripke models $\cdot$ Gödel-Dummett logic $\cdot$ Inter-definability of propositional connectives

\subsection{Introduction and Preliminaries}

Intuitionism grew out of some of the philosophical ideas of its founding father, Luitzen Egbertus Jan Brouwer (see e.g. Brouwer 1913); what is known nowadays as intuitionistic logic is a formalization given by his student Heyting (1930). Kripke models (originating from Kripke 1959) provided an interesting mathematical interpretation for this formalization. Let us review some preliminaries about these models:

Dedicated to Professor MOHAMMAD ARDESHIR with high appreciation and admiration.

\footnotetext{
S. Salehi $(\varangle)$

Research Institute for Fundamental Sciences, University of Tabriz, 29 Bahman Boulevard, 51666-16471, Tabriz, Iran e-mail: root@SaeedSalehi.ir URL: http://www.SaeedSalehi.ir/ 


\section{Definition 1 (Kripke Frames)}

A Kripke frame is a partially ordered set; i.e., an ordered pair $\langle K, \succcurlyeq\rangle$ where $\succcurlyeq \subseteq K^{2}$ is a reflexive, transitive and anti-symmetric binary relation on $K$.

\section{Definition 2 (Atoms, Formulas, Languages)}

Let At be the set of all the propositional atoms; atoms are usually denoted by letters $\mathfrak{p}$ or $\mathfrak{q}$. Let $T$ denote the verum (truth) constant.

The language of propositional logics studied here is $\mathscr{L}=\{\neg, \wedge, \vee, \rightarrow, \top\}$.

For any $A \subseteq$ At and $B \subseteq \mathscr{L}$, the set of all the formulas constructed from $A$ by means of $B$ is denoted by $\mathcal{L}(B, A)$.

Let $\mathrm{Fm}$ denote the set of all the formulas; i.e., $\mathcal{L}(\mathscr{L}, \mathrm{At})$.

Definition 3 (Kripke Models)

A Kripke model is a triple $\mathcal{K}=\langle K, \succcurlyeq, \Vdash\rangle$, where $\langle K, \succcurlyeq\rangle$ is a Kripke frame equipped with a persistent binary (satisfaction) relation $\Vdash \subseteq K \times A t$; persistency (of the relation $\Vdash$ with respect to $\succcurlyeq$ ) means that for all $k, k^{\prime} \in K$ and $\mathfrak{p} \in A t$, if $k^{\prime} \succcurlyeq k \Vdash \mathfrak{p}$ then $k^{\prime} \Vdash \mathfrak{p}$.

The satisfaction relation can be extended to all the (propositional) formulas, i.e., to $\Vdash \subseteq K \times \mathrm{Fm}$, as follows:

○ $k \Vdash T$.

$\circ k \Vdash(\varphi \wedge \psi) \Longleftrightarrow k \Vdash \varphi$ and $k \Vdash \psi$.

$\circ k \Vdash(\varphi \vee \psi) \Longleftrightarrow k \Vdash \varphi$ or $k \Vdash \psi$.

$\circ k \Vdash(\neg \varphi) \Longleftrightarrow \forall k^{\prime} \succcurlyeq k\left(k^{\prime} \nVdash \varphi\right)$.

$\circ k \Vdash(\varphi \rightarrow \psi) \Longleftrightarrow \forall k^{\prime} \succcurlyeq k\left(k^{\prime} \Vdash \varphi \Rightarrow k^{\prime} \Vdash \psi\right)$.

Remark 1 (On Persistency and its Converse)

It can be shown that the persistency conditions is inherited by the formulas; i.e., for any $k, k^{\prime} \in K$ in any Kripke model $\mathcal{K}=\langle K, \succcurlyeq, \Vdash\rangle$ and for any formula $\varphi$, if $k^{\prime} \succcurlyeq k \Vdash \varphi$ then $k^{\prime} \Vdash \varphi$.

Obviously, the converse may not hold $\left(k^{\prime} \Vdash \psi\right.$ and $k^{\prime} \succcurlyeq k$ do not necessarily imply that $k \Vdash \psi$ ); however, a partial converse holds for negated formulas:

if $k^{\prime} \succcurlyeq k$ and $k^{\prime} \Vdash \neg \varphi$, then $k \nVdash \varphi$.

By the soundness and completeness of the intuitionistic propositional logic (IPL) with respect to finite Kripke models, the tautologies of IPL are the formulas (in Fm) that are satisfied in all the elements of any finite Kripke model. A super-intuitionistic and sub-classical logic is the so-called Gödel-Dummett logic (see Dummett 1959), whose tautologies are the formulas that are satisfied in all the elements of all the connected finite Kripke models. A kind of Kripke model theoretic characterization for this logic is given in Safari and Salehi (2018).

\section{Definition 4 (Connectivity)}

A binary relation $R \subseteq K \times K$ is called connected, when for any $k, k^{\prime}, k^{\prime \prime} \in K$, if $k^{\prime} \succcurlyeq k$ and $k^{\prime \prime} \succcurlyeq k$, then we have either $k^{\prime} \succcurlyeq k^{\prime \prime}$ or $k^{\prime \prime} \succcurlyeq k^{\prime}$ (cf. Švejdar and Bendová 2000). 
The logic IPL is perhaps the most famous non-classical logic. A natural question (that according to Gödel 1932 was asked by his supervisor Hans Hahn) was whether IPL is a finitely many valued logic or not. Gödel (1932) showed that IPL is not finitely many valued. Jaśkowski (1936) showed that IPL is indeed a countably (infinite) many valued logic. In Sect. 11.2 we give alternative proofs for these theorems by using models of Kripke (1959). Gödel's proof gave birth to an intermediate logic, that today is called the Gödel-Dummett logic (GDL). Finally, in Sect. 11.3 we study the problem of inter-definability of propositional connectives in GDL and IPL.

\section{2 $\omega$-Many Values for Intuitionistic Propositional Logic}

Let us begin with a formal definition of a many-valued logic. Throughout the paper, we are dealing with propositional logics only.

Definition 5 (Many-Valued Logics)

A many-valued logic is $\langle\mathscr{V}, \tau, \sim, \Lambda, \mathrm{V}, \Rightarrow\rangle$, where $\mathscr{V}$ is a set of values with a designated element $\tau \in \mathscr{V}$ (interpreted as the truth) and the functions $\backsim: \mathscr{V} \rightarrow \mathscr{V}$, $\Lambda: \mathscr{V}^{2} \rightarrow \mathscr{V}, \mathrm{V}: \mathscr{V}^{2} \rightarrow \mathscr{V}$, and $\Rightarrow: \mathscr{V}^{2} \rightarrow \mathscr{V}$ constitute a truth table on $\mathscr{V}$

A valuation function is any mapping $\nu$ : At $\rightarrow \mathscr{V}$, which can be extended to all the formulas, denoted also by $\boldsymbol{\nu}: \mathrm{Fm} \rightarrow \mathscr{V}$, as follows:

○ $\nu(\neg \varphi)=\sim \nu(\varphi)$.

$\circ \boldsymbol{\nu}(\varphi \wedge \psi)=\boldsymbol{\nu}(\varphi) \Lambda \nu(\psi)$.

○ $\boldsymbol{\nu}(\varphi \vee \psi)=\boldsymbol{\nu}(\varphi) \mathrm{V} \boldsymbol{\nu}(\psi)$.

○ $\boldsymbol{\nu}(\varphi \rightarrow \psi)=\boldsymbol{\nu}(\varphi) \Rightarrow \boldsymbol{\nu}(\psi)$.

A formula $\theta$ is called tautology, when it is mapped to the designated value under any valuation function; i.e., $\nu(\theta)=\tau$ for any valuation $\nu$.

Theorem 1 appears in Safari (2017) and Safari and Salehi (2019). In the following, the disjunction operation $(\vee)$ is assumed to be commutative and associative.

Lemma 1 (A Tautology in $n$-Valued Logics)

For any $n>1$, the formula $W_{i<j \leqslant n}\left(\mathfrak{p}_{i} \rightarrow \mathfrak{p}_{\mathfrak{j}}\right)$ is a tautology in any $n$-valued logic in which the formula $(\mathfrak{p} \rightarrow \mathfrak{p}) \vee \mathfrak{q}$ is a tautology.

Proof In an $n$-valued logic, the $n+1$ atoms $\left\{\mathfrak{p}_{0}, \mathfrak{p}_{1}, \ldots, \mathfrak{p}_{n}\right\}$ can take $n$ values. So, under a valuation function, there should exist some $i<j \leqslant n$ such that $\mathfrak{p}_{i}$ and $\mathfrak{p}_{j}$ take the same value, by the Pigeonhole Principle. Since $(\mathfrak{p} \rightarrow \mathfrak{p}) \vee \mathfrak{q}$ is a tautology, then the formula $\mathbb{W}_{i<j \leqslant n}\left(\mathfrak{p}_{i} \rightarrow \mathfrak{p}_{\mathfrak{j}}\right)$ should be mapped to the designated value by all the valuation functions.

The lemma implies that the formula $(A \rightarrow B) \vee(A \rightarrow C) \vee(B \rightarrow C)$ is a tautology in the classical propositional logic; this formula is not a tautology in the intuitionistic (or even Gödel-Dummett) propositional logic. 
Theorem 1 (Gödel 1932: IPL Is Not Finitely Many Valued)

Intuitionistic propositional logic is not finitely many valued.

Proof By Lemma 1 it suffices to show that for any $n>1, \mathbb{W}_{i<j \leqslant n}\left(\mathfrak{p}_{i} \rightarrow \mathfrak{p}_{\mathfrak{j}}\right)$ is not a tautology in IPL. Consider the Kripke model $\mathcal{K}=\langle K, \succcurlyeq, \Vdash\rangle$ with

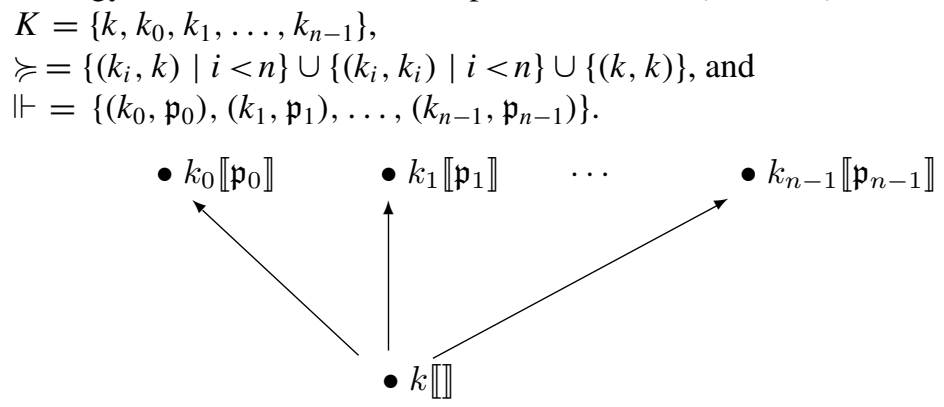

For any $i<n$ we have $k_{i} \Vdash \mathfrak{p}_{i}$, and also $k_{i} \nVdash \mathfrak{p}_{j}$ for any $j>i$. So, $k_{i} \nVdash \mathfrak{p}_{i} \rightarrow \mathfrak{p}_{j}$ for any $i<j \leqslant n$; which implies that $k \nVdash W_{i<j \leqslant n}\left(\mathfrak{p}_{i} \rightarrow \mathfrak{p}_{\mathfrak{j}}\right)$.

The rest of this section is devoted to proving Jaśkowski's result (Theorem 2) that IPL is a countably infinite many valued logic.

\section{Definition 6 (Monotone Functions)}

For a Kripke frame $(K, \succcurlyeq)$, a function $f: K \rightarrow\{0,1\}$ is called monotone, when for any $k, k^{\prime} \in K$, if $k^{\prime} \succcurlyeq k$, then $f\left(k^{\prime}\right) \geqslant f(k)$. We indicate the monotonicity of $f$ by writing $f:(K, \succcurlyeq) \rightarrow\{0,1\}$.

Example $1\left(\mathfrak{f}_{\mathcal{K}}^{\psi}\right)$

For any Kripke model $\mathcal{K}=(K, \succcurlyeq, \Vdash)$ and any formula $\psi$, the function

$$
\mathfrak{f}_{\mathcal{K}}^{\psi}: K \rightarrow\{0,1\}, \quad \mathfrak{f}_{\mathcal{K}}^{\psi}(k)= \begin{cases}1 & \text { if } k \Vdash \psi \\ 0 & \text { if } k \nVdash \psi\end{cases}
$$

is monotone.

Definition $7 \quad(\sim, \Lambda, \mathrm{V}$ and $\Rightarrow)$

For a Kripke frame $(K, \succcurlyeq)$ and monotone functions $f, g:(K, \succcurlyeq) \rightarrow\{0,1\}$, let

$\sim f: K \rightarrow\{0,1\}$ be defined by $(\sim f)(k)=\left\{\begin{array}{ll}1 & \text { if } \forall k^{\prime} \succcurlyeq k\left(f\left(k^{\prime}\right)=0\right) \\ 0 & \text { if } \exists k^{\prime} \succcurlyeq k\left(f\left(k^{\prime}\right)=1\right)\end{array}\right.$,

$f \Lambda g: K \rightarrow\{0,1\}$ be defined by $(f \Lambda g)(k)=\min \{f(k), g(k)\}$,

$f \mathrm{~V} g: K \rightarrow\{0,1\}$ be defined by $(f \mathrm{~V} g)(k)=\max \{f(k), g(k)\}$,

$f \Rightarrow g: K \rightarrow\{0,1\}$ be defined by

for all $k \in K$.

$$
(f \Rightarrow g)(k)=\left\{\begin{array}{ll}
1 & \text { if } \forall k^{\prime} \succcurlyeq k\left(f\left(k^{\prime}\right)=1 \Rightarrow g\left(k^{\prime}\right)=1\right) \\
0 & \text { if } \exists k^{\prime} \succcurlyeq k\left(f\left(k^{\prime}\right)=1 \& g\left(k^{\prime}\right)=0\right)
\end{array},\right.
$$




\section{Definition 8 (Constant Functions)}

Let $\mathbf{1}_{K}: K \rightarrow\{0,1\}$ be the constant 1 function, i.e., $\mathbf{1}_{K}(k)=1$ for all $k \in K$; and let $\mathbf{0}_{K}: K \rightarrow\{0,1\}$ be the constant 0 function: $\mathbf{0}_{K}(k)=0$ for all $k \in K$.

It is easy to see that the functions $\mathbf{1}_{K}$ and $\mathbf{0}_{K}$ obey the rules of the classical propositional logic with the operations $\backsim, \Lambda, \mathrm{V}$ and $\Rightarrow$. For example, $\left(\sim \mathbf{1}_{K}\right)=\mathbf{0}_{K}$, $\left(\mathbf{1}_{K} \Lambda \mathbf{1}_{K}\right)=\mathbf{1}_{K},\left(\mathbf{0}_{K} \mathrm{~V} \mathbf{1}_{K}\right)=\mathbf{1}_{K}$ and $\left(\mathbf{1}_{K} \Rightarrow \mathbf{0}_{K}\right)=\mathbf{0}_{K}$. We omit the proof of the following straightforward observation.

Lemma 2 (Monotonicity of $\mathbf{1}_{K}, \mathbf{0}_{K}, \sim f, f \Lambda g, f \mathrm{~V} g$ and $f \Rightarrow g$ )

For any Kripke frame $(K, \succcurlyeq)$, the constant functions $\mathbf{1}_{K}$ and $\mathbf{0}_{K}$ are monotone, and if $f, g:(K, \succcurlyeq) \rightarrow\{0,1\}$ are monotone, then so are $\sim f, f \Lambda g, f \mathrm{~V} g$ and $f \Rightarrow g$. $\square$

Finally, we can provide the following countably many values for IPL:

Definition 9 (Countably Many Values for IPL)

Enumerate all the finite Kripke frames as $\left(K_{0}, \succcurlyeq_{0}\right),\left(K_{1}, \succcurlyeq_{1}\right),\left(K_{2}, \succcurlyeq_{2}\right), \ldots$, where $K_{n} \subset \mathbb{N}$ for all $n \in \mathbb{N}$. Let

$\mathscr{V}=\left\{\left\langle f_{0}, f_{1}, f_{2}, \ldots\right\rangle \mid \forall n\left[f_{n}:\left(K_{n}, \succcurlyeq_{n}\right) \rightarrow\{0,1\}\right] \&\right.$

$\exists N \in \mathbb{N}\left[\left(\forall n \geqslant N f_{n}=\mathbf{1}_{K_{n}}\right)\right.$ or $\left.\left.\left(\forall n \geqslant N f_{n}=\mathbf{0}_{K_{n}}\right)\right]\right\}$.

In the other words, the set of values $\mathscr{V}$ consists of all the sequences $\left\langle f_{0}, f_{1}, f_{2}, \ldots\right\rangle$ such that for each $n, f_{n}$ is a monotone function on $\left(K_{n}, \succcurlyeq_{n}\right)$, and the sequences are ultimately constant (from a step onward, $f_{n}$ 's are either all $\mathbf{1}_{K_{n}}$ or all $\mathbf{0}_{K_{n}}$ ).

Let $\boldsymbol{\tau}=\left\langle\mathbf{1}_{K_{0}}, \mathbf{1}_{K_{1}}, \mathbf{1}_{K_{2}}, \ldots\right\rangle$ be the designated element (for truth).

For $\mathfrak{f}=\left\langle f_{0}, f_{1}, f_{2}, \ldots\right\rangle \in \mathscr{V}$ and $\mathfrak{g}=\left\langle g_{0}, g_{1}, g_{2}, \ldots\right\rangle \in \mathscr{V}$, let (cf. Definition 7)

$\sim \mathfrak{f}=\left\langle\sim f_{0}, \sim f_{1}, \sim f_{2}, \ldots\right\rangle$,

$\mathfrak{f} \Lambda \mathfrak{g}=\left\langle f_{0} \Lambda g_{0}, f_{1} \Lambda g_{1}, f_{2} \Lambda g_{2}, \ldots\right\rangle$,

$\mathfrak{f} \mathrm{V} \mathfrak{g}=\left\langle f_{0} \mathrm{~V} g_{0}, f_{1} \mathrm{~V} g_{1}, f_{2} \mathrm{~V} g_{2}, \ldots\right\rangle$, and

$\mathfrak{f} \Rightarrow \mathfrak{g}=\left\langle f_{0} \Rightarrow g_{0}, f_{1} \Rightarrow g_{1}, f_{2} \Rightarrow g_{2}, \ldots\right\rangle$.

It can be immediately seen that $\mathscr{V}$ is a countable set, and Lemma 2 implies that $\mathscr{V}$ is closed under the operations $\backsim, \Lambda, \mathrm{V}$ and $\Rightarrow$. Before proving the main theorem, we make a further definition and prove an auxiliary lemma.

Definition $10\left(\left\langle\langle\alpha\rangle_{n}, \Vdash_{n}^{\nu}\right.\right.$ and $\left.\nu_{m}^{\Vdash}\right)$

For a sequence $\alpha$, let $\langle\langle\alpha\rangle\rangle_{n}$ denote its $n$-th element (if any), for any $n \in \mathbb{N}$.

(1) Let a valuation $\nu:$ At $\rightarrow \mathscr{V}$ be given. The satisfaction relation $\Vdash_{n}^{\nu}$ is defined on any finite Kripke frame $\left(K_{n}, \succcurlyeq_{n}\right)$, with $K_{n} \subset \mathbb{N}$ (see Definition 9), by the following for any atom $\mathfrak{p} \in$ At and any $k \in K_{n}: k \Vdash_{n}^{\nu} \mathfrak{p} \Longleftrightarrow\left\langle\langle\boldsymbol{\nu}(\mathfrak{p})\rangle_{n}(k)=1\right.$.

(2) Let a Kripke model $\mathcal{K}=\left(K_{m}, \succcurlyeq_{m}, \Vdash\right)$ on the Kripke frame $\left(K_{m}, \succcurlyeq_{m}\right)$ be given (see Definition 9). Define the valuation $\nu_{m}^{\Vdash}$ by

$$
\boldsymbol{\nu}_{m}^{\Vdash}(\mathfrak{p})=\left\langle\mathbf{1}_{K_{0}}, \ldots, \mathbf{1}_{K_{m-1}}, \mathfrak{f}_{\mathcal{K}}^{\mathfrak{p}}, \mathbf{1}_{K_{m+1}}, \ldots\right\rangle
$$

for any $\mathfrak{p} \in$ At, where $\mathfrak{f}_{\mathcal{K}}^{\mathfrak{p}}: K_{m} \rightarrow\{0,1\}$ is the function that was defined in Example 1: $\mathfrak{f}_{\mathcal{K}}^{\mathfrak{p}}(k)=1$ if $k \Vdash \mathfrak{p}$, and $\mathfrak{f}_{\mathcal{K}}^{\mathfrak{p}}(k)=0$ if $k \nVdash \mathfrak{p}$, for any $k \in K_{m}$. 
It is clear that the relation $\Vdash_{n}^{\nu} \subseteq K_{n} \times$ At is persistent.

Lemma 3 (On $\Vdash_{n}^{\nu}$ and $\nu_{m}^{\Vdash}$ )

(1) Let a valuation $\nu:$ At $\rightarrow \mathscr{V}$ be given, and the satisfaction relation $\vdash_{n}^{\nu}$ be defined on $\left(K_{n}, \succcurlyeq_{n}\right)$ as in Definition 10. Then for any formula $\varphi \in$ Fm and any $k \in K_{n}$, we have $k \Vdash_{n}^{\nu} \varphi \Longleftrightarrow\langle\langle\nu(\varphi)\rangle\rangle_{n}(k)=1$.

(2) Let a Kripke model $\mathcal{K}=\left(K_{m}, \succcurlyeq_{m}, \Vdash\right)$ be given on the frame $\left(K_{m}, \succcurlyeq_{m}\right)$, and the valuation $\nu_{m}^{\Vdash}$ be defined as in Definition 10. Then for any formula $\varphi \in \mathrm{Fm}$ and any $k \in K_{m}$, we have $k \nVdash \varphi \Longleftrightarrow\left\langle\left\langle\boldsymbol{\nu}_{m}^{\Vdash}(\varphi)\right\rangle_{m}(k)=0\right.$.

Proof Both assertions can be proved by induction on $\varphi$. They are clear for $\varphi=\top$ and hold for atomic $\varphi \in$ At by Definition 10. The inductive cases follow immediately from Definitions 3, 5, 7, and 9.

Theorem 2 (Jaśkowski 1936: IPL Is Countably Many Valued) Intuitionistic propositional logic is countably infinite many valued.

Proof We show that a formula $\varphi \in$ Fm is satisfied in all the elements of all the finite Kripke models if and only if it is mapped to the designated element under all the valuation functions:

(1) If $\varphi$ is satisfied in any element of any finite Kripke model, then for any valuation $\boldsymbol{\nu}$ by Lemma 3(1) we have $\left\langle\langle\boldsymbol{\nu}(\varphi)\rangle_{n}=\mathbf{1}_{K_{n}}\right.$ for any $n \in \mathbb{N}$, so $\boldsymbol{\nu}(\varphi)=\boldsymbol{\tau}$.

(2) If $\varphi$ is not satisfied in some element of some finite Kripke model, then for some $m \in \mathbb{N}$ there is a Kripke model $\mathcal{K}=\left(K_{m}, \succcurlyeq_{m}, \Vdash\right)$ such that $\mathbb{k} \nVdash \varphi$ for some $\mathbb{k} \in K_{m}$. So, by Lemma 3(2) we have $\left\langle\left\langle\nu_{m}^{\Vdash}(\varphi)\right\rangle\right\rangle_{m}(\mathbb{k})=0$, thus $\nu_{m}^{\Vdash}(\varphi) \neq \tau$.

\subsection{Propositional Connectives Inside Gödel-Dummett Logic}

In classical propositional logic (which is a two-valued logic), all the connectives can be defined by (the so-called complete set of connectives) $\{\neg, \wedge\},\{\neg, \vee\}$ or $\{\neg, \rightarrow\}$ only. In this last section we will see that no propositional connective is definable from the others in IPL, and in GDL only the disjunction operation $(\vee)$ can be defined by the conjunction $(\wedge)$ and implication $(\rightarrow)$ operations. Most of these facts are already known (they appear in e.g. Safari and Salehi 2019 and Švejdar and Bendová 2000). Theorem 3 is from Švejdar and Bendová (2000) with a slightly different proof; Theorem 4 is from Švejdar and Bendová (2000) with the same proof. All of our proofs are Kripke model theoretic, as usual.

Theorem $3(\wedge$ Is Not Definable From the Others in GDL)

In Gödel-Dummett Logic, the conjunction connective $(\wedge)$ is not definable from the other propositional connectives.

Proof Consider the Kripke model $\mathcal{K}=\langle K, \succcurlyeq, \Vdash\rangle$ where $K=\{a, b, c\}, \succcurlyeq$ is the reflexive closure of $\{(a, b),(c, b)\}$, and $\Vdash=\{(a, \mathfrak{p}),(b, \mathfrak{p}),(b, \mathfrak{q}),(c, \mathfrak{q})\}$, for atoms $\mathfrak{p}, \mathfrak{q} \in$ At. 


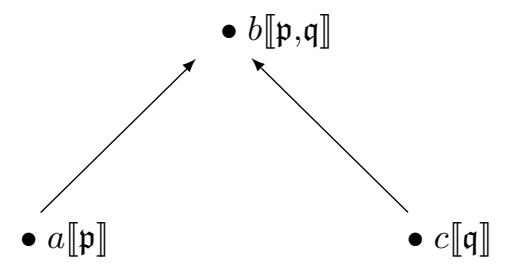

We show that for all formulas $\theta \in \mathcal{L}(\neg, \vee, \rightarrow, \top, \mathfrak{p}, \mathfrak{q})$ we have:

(*) $\quad b \Vdash \theta \Longrightarrow a \Vdash \theta$ or $c \Vdash \theta$.

This will prove the desired conclusion, since $b \Vdash \mathfrak{p} \wedge \mathfrak{q}$ but $a, c \nVdash \mathfrak{p} \wedge \mathfrak{q}$, and so $\mathfrak{p} \wedge \mathfrak{q}$ cannot belong to $\mathcal{L}(\neg, \vee, \rightarrow, \top, \mathfrak{p}, \mathfrak{q})$. We prove $(*)$ by induction on $\theta$. The cases of $\theta=\top, \mathfrak{p}, \mathfrak{q}$ are trivial, and the induction step of $\neg \varphi$ follows from Remark 1, and the case of $\varphi \vee \psi$ is rather easy. So, only the non-trivial case of $\theta=\varphi \rightarrow \psi$ remains. Suppose that $(*)$ holds for $\varphi$ and $\psi$, and assume (for the sake of a contradiction) that $b \Vdash \varphi \rightarrow \psi$ but $a, c \nVdash \varphi \rightarrow \psi$. So, $a \Vdash \varphi$ and $a \nVdash \psi$; and also $c \Vdash \varphi$ and $c \nVdash \psi$. Whence, by persistency, we should have also $b \Vdash \varphi$, thus $b \Vdash \psi$. So, by the induction hypothesis $(*$ for $\theta=\psi$ ) we should have either $a \Vdash \psi$ or $c \Vdash \psi$; a contradiction.

Theorem $4(\rightarrow$ Is Not Definable From the Others in GDL)

In Gödel-Dummett Logic, the implication connective $(\rightarrow)$ is not definable from the other propositional connectives.

Proof For the Kripke model $\mathcal{K}=\langle K, \succcurlyeq, \Vdash\rangle$, where $K=\{a, b, c\}, \succcurlyeq$ is the reflexive closure of $\{(a, b),(c, b)\}$, and $\Vdash=\{(a, \mathfrak{p}),(b, \mathfrak{p}),(b, \mathfrak{q})\}$, for $\mathfrak{p}, \mathfrak{q} \in A t$,

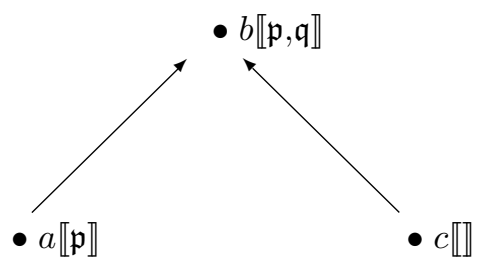

we show that for all the formulas $\theta \in \mathcal{L}(\neg, \vee, \wedge, \top, \mathfrak{p}, \mathfrak{q})$, the following holds:

(*) $\quad b, c \Vdash \theta \Longrightarrow a \Vdash \theta$.

This completes the proof since $b, c \Vdash \mathfrak{p} \rightarrow \mathfrak{q}$ but $a \nVdash \mathfrak{p} \rightarrow \mathfrak{q}$ (by $a \Vdash \mathfrak{p}, a \nVdash \mathfrak{q}$ ); thus we have $(\mathfrak{p} \rightarrow \mathfrak{q}) \notin \mathcal{L}(\neg, \vee, \wedge, \top, \mathfrak{p}, \mathfrak{q})$. The proof of $(*)$ is by induction on $\theta$; the only non-trivial cases to consider are $\theta=\varphi \vee \psi$ and $\theta=\varphi \wedge \psi$. Suppose that $(*)$ holds for $\varphi$ and $\psi$; and that $b, c \Vdash \varphi \vee \psi$. Then we have either $c \Vdash \varphi$ or $c \Vdash \psi$; by the persistency, the former implies $b \Vdash \varphi$ and the latter $b \Vdash \psi$. So, in either case by the induction hypothesis we have $a \Vdash \varphi \vee \psi$. The case of $\theta=\varphi \wedge \psi$ is even simpler.

The following has been known for a long time; see e.g. Dummett (1959).

Theorem 5 ( $\vee$ Is Definable From $\wedge, \rightarrow$ in GDL)

In Gödel-Dummett Logic, the disjunction connective $(\vee)$ is definable from some other propositional connectives. 
Proof It is rather easy to see that IPL $\Vdash(\mathfrak{p} \vee \mathfrak{q}) \longrightarrow[(\mathfrak{p} \rightarrow \mathfrak{q}) \rightarrow \mathfrak{q}] \wedge[(\mathfrak{q} \rightarrow \mathfrak{p}) \rightarrow \mathfrak{p}]$. Now, we show that GDL $\Vdash[(\mathfrak{p} \rightarrow \mathfrak{q}) \rightarrow \mathfrak{q}] \wedge[(\mathfrak{q} \rightarrow \mathfrak{p}) \rightarrow \mathfrak{p}] \longrightarrow(\mathfrak{p} \vee \mathfrak{q})$ holds. Take an arbitrary connected Kripke model $\mathcal{K}=\langle K, \succcurlyeq, \Vdash\rangle$, and suppose that for an arbitrary $a \in K$ we have $a \Vdash[(\mathfrak{p} \rightarrow \mathfrak{q}) \rightarrow \mathfrak{q}] \wedge[(\mathfrak{q} \rightarrow \mathfrak{p}) \rightarrow \mathfrak{p}]$. We show that $a \Vdash \mathfrak{p} \vee \mathfrak{q}$. Assume not; then $a \nVdash \mathfrak{p}, \mathfrak{q}$. Therefore, $a \nVdash(\mathfrak{p} \rightarrow \mathfrak{q})$ and $a \nVdash(\mathfrak{q} \rightarrow \mathfrak{p})$, by $a \Vdash[(\mathfrak{p} \rightarrow \mathfrak{q}) \rightarrow \mathfrak{q}]$ and $a \Vdash[(\mathfrak{q} \rightarrow \mathfrak{p}) \rightarrow \mathfrak{p}]$, respectively. So, there are some $b, c \in K$ with $b, c \succcurlyeq a$ such that $b \Vdash \mathfrak{p}, b \nVdash \mathfrak{q}, c \Vdash \mathfrak{q}$, and $c \nVdash \mathfrak{p}$.

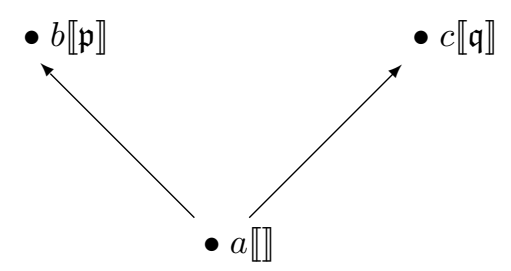

By the connectivity of $\succcurlyeq$, we should have either $b \succcurlyeq c$ or $c \succcurlyeq b$. Both cases lead to a contradiction, by the persistency condition. So, the following equivalence

$$
(\mathfrak{p} \vee \mathfrak{q}) \equiv[(\mathfrak{p} \rightarrow \mathfrak{q}) \rightarrow \mathfrak{q}] \wedge[(\mathfrak{q} \rightarrow \mathfrak{p}) \rightarrow \mathfrak{p}]
$$

holds in GDL.

The fact of the matter is that $(\mathfrak{p} \vee \mathfrak{q}) \equiv[(\mathfrak{p} \rightarrow \mathfrak{q}) \rightarrow \mathfrak{q}] \wedge[(\mathfrak{q} \rightarrow \mathfrak{p}) \rightarrow \mathfrak{p}]$ is the only non-trivial equivalence relation between the propositional connectives in GDL. The first half of the following theorem was proved in Safari and Salehi (2019).

Theorem 6 (In GDL $\vee$ Is Not Definable Without Both $\wedge, \rightarrow$ )

In Gödel-Dummett Logic, disjunction $(\vee)$ is not definable from the other propositional connectives, unless both the conjunction and the implication connectives are present. In the other words, $\vee$ is definable neither from $\{\neg, \rightarrow, \top\}$ nor from $\{\neg, \wedge, \top\}$.

Proof Take the Kripke model $\mathcal{K}=\langle K, \succcurlyeq, \Vdash\rangle$ with $K=\{a, b, c, d\}, \succcurlyeq=$ the reflexive closure of $\{(a, b),(c, d)\}$, and $\Vdash=\{(b, \mathfrak{p}),(d, \mathfrak{q})\}$, for $\mathfrak{p}, \mathfrak{q} \in A t$.

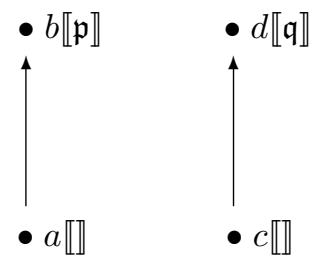

We show that for all $\theta \in \mathcal{L}(\neg, \rightarrow, \top, \mathfrak{p}, \mathfrak{q})$ we have

(*) $\quad b, d \Vdash \theta \Longrightarrow a \Vdash \theta$ or $c \Vdash \theta$.

Since $b, d \Vdash \mathfrak{p} \vee \mathfrak{q}$ but $a, c \nVdash \mathfrak{p} \vee \mathfrak{q}$, then it follows that $\mathfrak{p} \vee \mathfrak{q} \notin \mathcal{L}(\neg, \rightarrow, \top, \mathfrak{p}, \mathfrak{q})$.

Now, $(*)$ can be proved by induction on $\theta$; the only non-trivial case is $\theta=\varphi \rightarrow \psi$. If

(*) holds for $\varphi$ and $\psi$, then if $b, d \Vdash \varphi \rightarrow \psi$ but $a \nVdash \varphi \rightarrow \psi$ and $c \nVdash \varphi \rightarrow \psi$, then we 
should have $a \Vdash \varphi$ and $a \nVdash \psi$, and also $c \Vdash \varphi$ and $c \nVdash \psi$. So, by persistency, $b \Vdash \varphi$ and $d \Vdash \varphi$; thus $b \Vdash \psi$ and $d \Vdash \psi$. So, by the induction hypothesis ( $*$ for $\theta=\psi$ ) we should have either $a \Vdash \psi$ or $c \Vdash \psi$; a contradiction.

Now, for proving $\mathfrak{p} \vee \mathfrak{q} \notin \mathcal{L}(\neg, \wedge, \top, \mathfrak{p}, \mathfrak{q})$, we show that for all the formulas $\theta$ in $\mathcal{L}(\neg, \wedge, \top, \mathfrak{p}, \mathfrak{q})$ we have

(†) $\quad b, d \Vdash \theta \Longrightarrow a, c \Vdash \theta$.

Trivially, ( ) holds for $\theta=\top, \mathfrak{p}, \mathfrak{q}$; so by Remark 1 it only suffices to show that ( $)$ holds for $\theta=\varphi \wedge \psi$, when it holds for $\varphi$ and $\psi$. Now, if $b, d \Vdash \varphi \wedge \psi$ then $b, d \Vdash \varphi$ and $b, d \Vdash \psi$; so the induction hypothesis ( $\ddagger$ for $\theta=\varphi, \psi$ ) implies that $a, c \Vdash \varphi$ and $a, c \Vdash \psi$, therefore $a, c \Vdash \varphi \wedge \psi$.

We end the paper with a Kripke model theoretic proof of a known fact.

Proposition 1 (No Connective Is Definable From the Others in IPL) In IPL, no propositional connective is definable from the others.

Proof By Theorems 3 and $4, \wedge$ and $\rightarrow$ are not definable from the other connectives even in GDL. The statement $\neg \mathfrak{p} \notin \mathcal{L}(\wedge, \vee, \rightarrow, \top, \mathfrak{p})$ can be easily verified by noting that all the operations on the righthand side are positive. So, it only remains to show that we have $\mathfrak{p} \vee \mathfrak{q} \notin \mathcal{L}(\neg, \wedge, \rightarrow, \top, \mathfrak{p}, \mathfrak{q})$ in IPL (cf. Theorem 5). Consider the Kripke model $\mathcal{K}=\langle K, \succcurlyeq, \Vdash\rangle$ with $K=\{a, b, c\}, \succcurlyeq=$ the reflexive closure of $\{(a, b),(a, c)\}$, and $\Vdash=\{(b, \mathfrak{p}),(c, \mathfrak{q})\}$, for $\mathfrak{p}, \mathfrak{q} \in$ At.

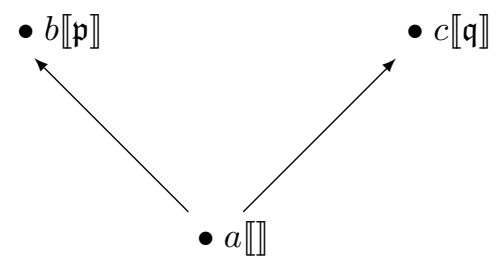

We show that for all formulas $\theta \in \mathcal{L}(\neg, \wedge, \rightarrow, \top, \mathfrak{p}, \mathfrak{q})$ we have:

(*) $\quad b, c \Vdash \theta \Longrightarrow a \Vdash \theta$.

This will prove the theorem, since $b, c \Vdash \mathfrak{p} \vee \mathfrak{q}$ but $a \nVdash \mathfrak{p} \vee \mathfrak{q}$, and so $\mathfrak{p} \vee \mathfrak{q}$ is not in $\mathcal{L}(\neg, \wedge, \rightarrow, \top, \mathfrak{p}, \mathfrak{q})$ in IPL. Indeed, $(*)$ can be proved by induction on $\theta$; for which we consider the case of $\theta=\varphi \rightarrow \psi$ only. So, suppose that $(*)$ holds for $\varphi$ and $\psi$ and that $b, c \Vdash \varphi \rightarrow \psi$ but $a \nVdash \varphi \rightarrow \psi$. Then we should have $a \Vdash \varphi$ and $a \nVdash \psi$; but by persistency we should have that $b, c \Vdash \varphi$, and so $b, c \Vdash \psi$ holds. Now, the induction hypothesis $(*$ for $\theta=\psi$ ) implies that $a \Vdash \psi$, a contradiction. 


\section{References}

Brouwer, L. E. J. (1913). Intuitionism and formalism. Bulletin of the American Mathematical Society, 20(2), 81-96. https://doi.org/10.1090/S0273-0979-99-00802-2.

Dummett, M. (1959). A propositional calculus with denumerable matrix. The Journal of Symbolic Logic, 24(2), 97-106. https://doi.org/10.2307/2964753.

Gödel, K. (1932). Zum Intuitionistischen Aussagenkalkül. Anzeiger Akademie der Wissenschaften Wien, 69, 65-66. Translated as: On the intuitionistic propositional calculus. In S. Feferman, et al. (Eds.) (1986). Kurt Gödel collected works (Volume I) Publications 1929-1936. (pp. 222-225). Oxford University Press.

Heyting, A. (1930). Die Formalen Regeln der Intuitionistischen Logik. Sitzungsberichte der Preussischen Akademie von Wissenschaften, Physikalisch Mathematische Klasse, 42-56. Die Formalen Regeln der Intuitionistischen Mathematik I, ibid. 57-71. II, ibid. 158-169.

Jaśkowski, S. (1936). Recherches sur le Systéme de la Logique Intuitioniste. Actes du Congrés International de Philosophie Scientifique, VI. Philosophie des Mathématiques, Actualités Scientifiques et Industrielles 393, Hermann \& C $\mathrm{C}^{\mathrm{ie}}$, Parsi, 58-61.

Kripke, S. A. (1959). A completeness theorem in modal logic. The Journal of Symbolic Logic, 24(1), 1-14. https://doi.org/10.2307/2964568.

Safari, P. (2017). Investigating Kripke semantics for fuzzy logics (in Persian). Ph.D. Dissertation, University of Tabriz. Under the Supervision of Saeed Salehi. https://bit.ly/3ivYGC2.

Safari, P., \& Salehi, S. (2018). Kripke semantics for fuzzy logics. Soft Computing, 22(3), 839-844. https://doi.org/10.1007/s00500-016-2387-4.

Safari, P., \& Salehi, S. (2019). Truth values and connectives in some non-classical logics. Journal of New Researches in Mathematics, 5(19), 31-36 (in Persian). https://bit.ly/2FpMC7f.

Švejdar, V., \& Bendová, K. (2000). On inter-expressibility of logical connectives in Gödel fuzzy logic. Soft Computing, 4(2), 103-105. https://doi.org/10.1007/s005000000036. 